\title{
植物CRISPR/Cas9多基因编辑载体构建和 突变分析的操作方法
}

曾栋昌 ${ }^{1 \dagger}$, 马兴亮 ${ }^{1,2 \dagger}$, 谢先荣 ${ }^{1 \dagger}$, 祝钦泷 ${ }^{1}$, 刘耀光 ${ }^{1^{*}}$

1. 华南农业大学生命科学学院, 亚热带农业生物资源保护与利用国家重点实验室, 广州 510642;

2. Seed and Developmental Biology Program, Global Institute for Food Security, University of Saskatchewan, Saskatoon, SK S7N 4J8, Canada $\dagger$ 同等贡献

* 联系人, E-mail: ygliu@scau.edu.cn

收稿日期：2018-04-08; 接受日期：2018-04-20; 网络发表日期：2018-06-21

广东省公益研究与能力建设项目(批准号: 2015B020201002)和转基因专项(批准号: 2016ZX08010001, 2016ZX08009002)资助

摘要 CRISPR/Cas9基因组编辑技术是植物基因功能研究与作物改良的有效工具. 为此, 本实验室开发了高效的 CRISPR/Cas9植物多基因编辑载体系统. 本载体系统包括 6 个双元载体和 12 个含有不同U3/U6启动子的 $\operatorname{sgRNA}$ 中 间载体，可满足对单子叶和双子叶植物的遗传转化以及不同抗生素篎选的要求，具有简便、高效，可同时对多基 因进行编辑的特点. 此外, 为了能更高效地应用基因组编辑技术, 还开发了一站式在线分析工具包CRISPR-GE. 为 方便研究人员利用CRISPR/Cas9系统进行植物基因组编辑, 本文提供了从靶点选择、CRISPR/Cas9多靶点双元载 体构建, 以及对靶点突变序列的测序分析等详细的操作方法, 以及常见的问题解答.

关键词 植物基因组编辑, CRISPR/Cas9, 定点突变, CRISPR-GE

基因组编辑技术能精确地修饰基因组中的特定位 点, 从而可很方便地获得新的具有重要价值的突变体. 基于成簇的规律间隔的短回文重复序列(clustered regularly interspaced short palindromic repeats, CRISPR)/ Cas9的基因组编辑技术以其高效，简便的优势迅速获 得了广泛应用，已成为植物以及作物的基础研究与遗 传改良的必不可少的工具 ${ }^{[1,2]}$.

在模式植物以及各种作物中，应用CRISPR/Cas9 基因编辑技术的实例越来越多 ${ }^{[3,4]}$. 本实验室开发了一 套用于植物的简便高效的多靶点CRISPR/Cas 9 基因编 辑载体系统 ${ }^{[5]}$, 可同时编辑植物基因组的多个靶位点.
目前，该载体系统已被国内外大量实验室利用，在植 物基因功能研究和作物性状遗传改良中发挥着重要 作用。

为了方便CRISPR/Cas9的靶位点设计, 载体构建, 以及突变靶点测序分析，本实验室还开发了配套的一 站式在线软件工具包CRISPR-GE (http://skl.scau.edu. $\mathrm{cn} /$ ). CRISPR-GE由 5 个独立和关联的子程序构成, 包 括从参考基因组下载任意区段序列的“seqDownload”, 进行靶点设计的“targetDesign”, 对潜在脱靶位点评估 的“offTarget”，载体sgRNA表达盒构建和靶点扩增测 序的引物设计的“primerDesign”, 以及对突变靶点测序

引用格式: 曾栋昌, 马兴亮, 谢先荣, 等. 植物CRISPR/Cas9多基因编辑载体构建和突变分析的操作方法. 中国科学: 生命科学, 2018, 48: 783-794 Zeng D C, Ma X L, Xie X R, et al. A protocol for CRISPR/Cas9-based multi-gene editing and sequence decoding of mutant sites in plants (in Chinese). Sci Sin Vitae, 2018, 48: 783-794, doi: 10.1360/N052018-00069 
文件解码的“DSDecodeM”[6-9]. 为了方便研究人员利用 CRISPR/Cas9载体系统进行植物基因组编辑，本文提 供了从靶点选择、CRISPR/Cas 9多靶点双元载体构 建、到对突变靶点进行测序分析等实验的详细操作 方法.

\section{1 材料}

\section{1 试剂}

Bsa I -HF (NEB, cat. no. R3535, 美国)和其他常用 的内切酶; Phanta Max Super-Fidelity DNA Polymerase (Vazyme, cat. no. P505-d1, 南京)或KOD-FX (TOYOBO, cat. no. KFX-101，上海)或KOD Plus (TOYOBO, cat. no. KOD-201); PCR产物纯化试剂盒(GenStar, cat. no.D205-01, 北京); T4 DNA连接酶(TaKaRa cat. no.2011A, 日本); ATP (Thermo Scientific, cat.no. $\mathrm{R} 0441$ ，美国); 去离子水 $\left(\mathrm{ddH}_{2} \mathrm{O}\right) ; \mathrm{TE}$ 缓冲溶液; LB, SOC等液体培养基; LB琼脂板培养基(氨芐青霉素抗 性 $100 \mu \mathrm{g} / \mathrm{mL}$, 卡那霉素抗性 $25 \mu \mathrm{g} / \mathrm{mL}$ ); 其他分子生物 学常用试剂.

\section{2 仪器与耗材}

超净工作台; 恒温培养箱; 细菌培养摇床; 台式离 心机; 凝胶电泳仪; 凝胶电泳槽; 凝胶成像仪; 金属浴 (或水浴锅); 移液器; PCR管; 悬浮式透析膜Millipore VSWP04700 (0.025 $\mu \mathrm{m}$ 孔径); 电激转化仪.

\section{3 载体}

整套载体的信息和图谱见表1和图1。这些载体可 以从本实验室索取, 也可以通过Addgene (https://www. addgene.org/)获取。载体的参考序列从 $\mathrm{NCBI}$ 的GenBank下载.

\section{4 引物及其搭配}

构建多个 sgRNA表达盒和分析引物见表 2 , 和第二 轮PCR扩增sgRNA表达盒的引物搭配使用原则见表3.

\section{$1.5 \mathrm{U} 3 / \mathrm{U} 6$ 启动子的选用和排列}

本载体系统有多个U3/U6启动选用. 本研究表明, 水稻来源的4种启动子 $(O s U 3, O s U 6 a, O s U 6 b, O s U 6 c)$ 在水稻表达 sgRNA 具有同等的编辑效率 ${ }^{[5]}$. 但是, 在构
建多靶点编辑载体时如果不同的靶点表达盒都使用同 一个启动子，就形成序列相同(只有 $20 \mathrm{bp}$ 靶点不同)的 启动子-sgRNA表达盒(约500 900 bp)的多串联重复, 增加了载体在农杆菌内发生同源重组结构变异的风 险. 因此建议使用不同的启动子, 参考图2的排列(可以 适当改变)构建多靶点编辑载体. 其中使用一个含 $L a c Z$ 的启动子可产生蓝色菌斑篎选阳性克隆.

对于构建双子叶植物编辑载体，可以使用拟南芥 (Arabidopsis thaliana) 来源的4种启动子 $(A t U 3 b, A t U 3 d$, $A t U 6-1, A t U 6-29)$, 参照图2进行排列. 但是, 由于AtU3d 很短(只有 $102 \mathrm{bp}$ ), 以此启动子构建的多个 $\operatorname{sgRNA}$ 表达 盒产生的串联重复载体在农杆菌也不会产生结构变 异，因此可以只使用AtU3d（和一个LacZ-AtU3d)构建 多靶点编辑载体.

\section{2 实验流程}

\subsection{CRISPR-GE软件智能靶点分析设计及引物自 动生成方法}

(1) 靶点设计. 打开CRISPR-GE网页(http://sk1. scau.edu.cn/), 进入靶点设计(targetDesign)子程序, 选 择对应的PAM类型以及靶向参考基因组. 在“Sequence submission”框中粘贴靶基因序列，或输入基因号信息 (和上下游序列长度). 点击“Submit”即可运行程序. 在 靶点设计的结果中可查看所有候选靶点的序列、位 置、正负链、 $\mathrm{GC}$ 含量、潜在的脱靶位等点和估值等 信息. “targetDesign”还对有连续 4 个或以上 $\mathrm{T}$ 碱基(可能 成为转录终止信号), 较低( $<30 \%)$ 的 GC含量, 和靶序列 与 sgRNA序列有 8 碱基以上配对(产生RNA茎-环结构) 的候选靶点判定为编辑效率可能较低的“坏”靶点而给 出警示(!或!!). 脱靶估值等于或大于 0.7 (或 0.6 )的靶点 的脱靶突变的风险较高, 尽可能不选用. 一般选择 GC 含量 $45 \%$ 70\%, 位置符合要求的靶点(正链和负链都可 以), 勾选后点击“primerDesign”跳转至“primerDesignV”, 可生成构建表达盒的引物.

（2）设计用于表达盒构建的引物. 如果不是以“targetDesign”设计目标基因组区的靶点, 而是已经确定了 靶点序列, 就进入 “primerDesign”, 在“primerDesign-V” 的“Target sequence of CRISPR/Cas9”框中粘贴20碱基 的靶点序列和PAM碱基，更多靶点需点击“Insert”. 然 后在“Promoter”选项选择相应的U3/U6启动子，在 


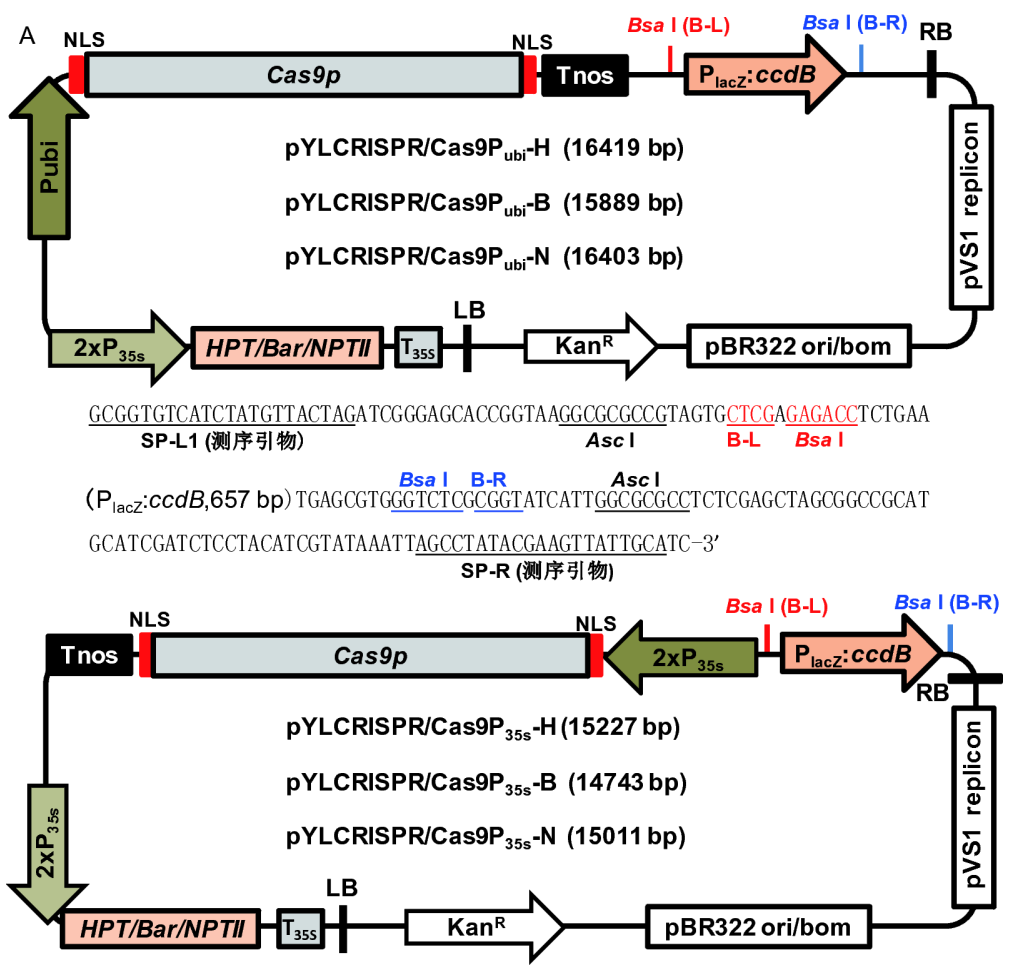

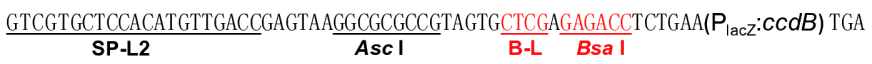

$$
\begin{aligned}
& \begin{array}{l}
\text { Bsa I B-R A AscI } \\
\text { GCGTGGTCTCGCGGTATCATTGGCGCGCCTCTCGAGCTAGCGGCCGCATGCATCGATCTCCTACATCGTATA }
\end{array} \\
& \text { AATTAGCCTATACGAAGTTATTGCATCT-3' }
\end{aligned}
$$

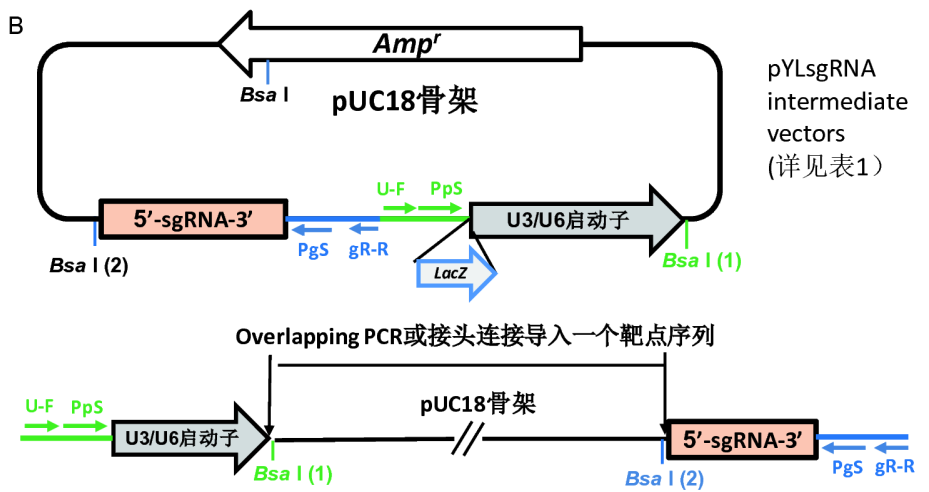

图 1 植物基因组编辑双元载体和sgRNA中间载体的结构

A: pYLCRISPR/Cas9双元载体结构. 本套双元载体骨架为pCAMBIA-1300; Cas9p为植物密码子优化的合成基因, 其两端加了核定位信号(nuclear localization signal, NLS). 这些双元载体质粒需要在含有基因型Lacl $I^{q}$ 的Escherichia coli菌株(如TOP10F')中繁殖; 该菌株LacI ${ }^{q}$ 基因型产生 的高水平阻碍蛋白可抑制由 $\mathrm{P}_{\mathrm{lacz}}$ 启动子控制的致死基因 $c c d B$ 的表达. pYLCRISPR/Cas $9 \mathrm{Pubi}-\mathrm{H},-\mathrm{N}$ ，-B载体中的Cas9p 由玉米泛素基因启动子 $\mathrm{P}_{\mathrm{ubi}}$ 驱动, 分别使用潮霉素抗性基因(HPT)、卡那霉素抗性基因(NPTII), 和除草剂抗性基因(Bar), 优先用于单子和大部分双子叶植物的基因组 编辑. 另外 3 个双元载体为以 $\mathrm{P}_{35 \mathrm{~s}}$ 启动子驱动 Cas $9 p$ 的 $\mathrm{pYLCRISPR} / \mathrm{Cas} 9 \mathrm{P}_{35 \mathrm{~s}} \mathrm{-H},-\mathrm{N},-\mathrm{B}(\mathrm{A})$, 可用于双子叶植物的基因组编辑. 这些双元载体的 $c c d B$ 基因 2 端各有 1 个IIs 型限制性内切酶 $B s a$ I位点, 酶切后产生非回文黏性末端B-L和B-R, 用于以“金门”(Golden Gate)克隆法 ${ }^{[10]}$ 将sgRNA表达 盒连接到载体; B: pYLgRNA中间载体结构. 载体中的sgRNA序列长76 bp (不含靶序列). 本系统有来源于水稻(Oryza sativa)的4个不同 snRNA 基因启动子 ( OsU3, Os U6a, OsU6b, OsU6c, 用于单子叶植物), 和来源于拟南芥的4个不同snRNA基因启动子( $A t U 3 b, A t U 3 d, A t U 6-1, A t U 6-29$, 用 于双子叶植物). 部分载体在U3或U6启动子上游含有 $L a c Z$ 基因(表1), 用于阳性克隆的篮选. Bsa I (1)和 $B s a$ I (2)位点用于将靶点双链接头连 接到sgRNA表达盒, 但以overlapping PCR方法就不需要Bsa I 酶切 
表 1 CRISPR/Cas9双元载体及含U3/U6 的sgRNA中间载体的基本信息 ${ }^{a)}$

\begin{tabular}{|c|c|c|c|c|}
\hline 载体名称 & 原核抗性 & 植物抗性基因 & GenBank登录号 & Addgene索取号 \\
\hline pYLCRISPR/Cas9Pubi-H & Kana & $H P T$ & KR029109 & 66187 \\
\hline pYLCRISPR/Cas9Pubi-N & Kana & NPTII & MG719602 & 106331 \\
\hline pYLCRISPR/Cas9Pubi-B & Kana & Bar & KR029110 & 66188 \\
\hline pYLCRISPR/Cas $9 \mathrm{P}_{35 \mathrm{~s}}-\mathrm{H}$ & Kana & $H P T$ & KR029111 & 66189 \\
\hline pYLCRISPR/Cas $9 \mathrm{P}_{35 \mathrm{~s}}-\mathrm{N}$ & Kana & NPTII & KR029112 & 66190 \\
\hline pYLCRISPR/Cas $9 \mathrm{P}_{35 \mathrm{~s}}-\mathrm{B}$ & Kana & Bar & KR029113 & 66191 \\
\hline pYLgRNA-OsU3 & Amp & - & KR029097 & 66192 \\
\hline pYLgRNA-OsU3/LacZ & Amp & - & KR029098 & 66193 \\
\hline pYLgRNA-OsU6a & Amp & - & KR029099 & 66194 \\
\hline pYLgRNA-OsU6a/LacZ & Amp & - & KR029100 & 66195 \\
\hline pYLgRNA-OsU6b & Amp & - & KR029101 & 66196 \\
\hline pYLgRNA-OsU6c & Amp & - & KR029102 & 66197 \\
\hline pYLgRNA-AtU3b & Amp & - & KR029103 & 66198 \\
\hline pYLgRNA-AtU3b/LacZ & Amp & - & KR029104 & 66199 \\
\hline pYLgRNA-AtU3d & Amp & - & KR029105 & 66200 \\
\hline pYLgRNA-AtU3d/LacZ ${ }^{*}$ & Amp & - & KR029106 & 66201 \\
\hline pYLgRNA-AtU6-1 & Amp & - & KR029107 & 66202 \\
\hline pYLgRNA-AtU6-29 & Amp & - & KR029108 & 66203 \\
\hline
\end{tabular}

a) *: 这些载体含有 $L a c Z$ 基因(图1B)

“Method”中选择引物设计的方法：双链接头连接扩增 法(Method 1)或Overlapping PCR法(Method 2). 之后点 击“Design”, 程序自动生成构建表达盒的引物. 由于不 同的U3/U6启动子具有不同的序列和转录起始碱基, 程序会根据选用的不同启动子，生成的引物的序列也 不同. 双链接头具有与 $B s a$ I 酶切 $\mathrm{sgRNA}$ 中间载体的 $B s a$ I (1)和 $B s a$ I (2)位点配对的黏性末端.

\section{2 菌种活化和质粒提取制备及其质量检测}

将选好的pYLCRISPR/Cas9质粒菌种(TOP10F')和 pYLgRNA-U3/U6质粒菌种(DH10B)分别在含有卡那 霉素 $(25 \mu \mathrm{g} / \mathrm{mL})$ 和氨苄青霉素 $(100 \mu \mathrm{g} / \mathrm{mL}) \quad \mathrm{LB}$ 平板培 养基划线培养过夜，挑取单菌落用LB液体培养，并用 质粒提取试剂盒提取质粒.

配制酶切反应检查质粒的纯度(易酶切表示纯度 高)和确认内切酶 $B s a$ I 的活性(此酶较容易失活):

(i ) 用 3 U Bsa I -HF切 300 ng pYLCRISPR/Cas9质 粒 $\left(15 \mu \mathrm{L}\right.$ 体系, $37^{\circ} \mathrm{C} 15 \mathrm{~min}$ ), 电泳检查(未切的等量质 粒为对照). (ii) 用 $\sim 3 \mathrm{U} B s a$ I -HF切 $100 \mathrm{ng}$ pYLgR-
NA-U3/U6 $\left(10 \mu \mathrm{L}\right.$ 体系, $37^{\circ} \mathrm{C} 15 \mathrm{~min}$ ), 电泳检查(未切 的等量质粒为对照). (iii) 如果显示以上 2 种反应的酶 切效率都低, 用 $3 \mathrm{U} \mathrm{Bsa}$ I - HF切 100 ng 高纯度的含 有氨茮青霉素抗性基因 $A m p^{r}$ 的质粒如 $\mathrm{pUC18} / 19\left(A m p^{r}\right.$ 含有 1 个 $B s a$ I 位点, 图1B), 以确认 $B s a$ I - HF的活性 是否正常.

\section{$2.3 \operatorname{sgRNA}$ 表达盒的构建}

在构建表达盒的方法中, 有两种构建方法可选, 方 法1: 靶点接头连接扩增法, 原理图解参考已发表的文 章及操作手册 ${ }^{[5,9]}$ ，实验操作本文也有介绍; 方法 2 : Overlapping PCR法, 原理参考图3A. 使用者可以在步 骤2.1 (2)中任意选择构建表达盒引物的方法以及对应 的构建sgRNA表达盒的方法

(1) Overlapping PCR法. 第一轮PCR. 此步骤的目 的是分别将靶点序列引入到U3/U6启动子下游和 sgRNA序列的上游(图3A).

$2 \times$ Phanta Max Buffer $7.5 \mu \mathrm{L}$

$10 \mathrm{mmol} / \mathrm{L}$ dNTPs Mix $0.25 \mu \mathrm{L}$ 
表 2 构建多个sgRNA表达盒的通用引物a)

\begin{tabular}{|c|c|c|}
\hline 靶位点 & 引物 & 序列 $\left(5^{\prime} \rightarrow 3^{\prime}\right)$ \\
\hline \multirow{2}{*}{$1^{\text {st }} \mathrm{PCR}$} & U-F & CTCCGTTTTACCTGTGGAATCG \\
\hline & gR-R & CGGAGGAAAATTCCATCCAC \\
\hline \multirow{2}{*}{$\begin{array}{l}2^{\text {nd }} \text { PCR } \\
\text { 位点B-R } \\
\text { 位点2 }\end{array}$} & Pps-R & TTCAGAGGTCTCTACCGACTAGTCACGCGTATGGAATCGGCAGCAAA \\
\hline & Pgs-2 & AGCGTGGGTCTCGTCAGGGTCCATCCACTCCAAGCTC \\
\hline \multirow{2}{*}{$\begin{array}{l}\text { 位点2 } \\
\text { 位点3 }\end{array}$} & Pps-2 & TTCAGAGGTCTCTCTGACACTGGAATCGGCAGCAAAGG \\
\hline & Pgs-3 & AGCGTGGGTCTCGTCTTCACTCCATCCACTCCAAGCTC \\
\hline \multirow{2}{*}{$\begin{array}{l}\text { 位点3 } \\
\text { 位点4 }\end{array}$} & Pps-3 & TTCAGAGGTCTCTAAGACTTTGGAATCGGCAGCAAAGG \\
\hline & Pgs-4 & AGCGTGGGTCTCGAGTCCTTTCCATCCACTCCAAGCTC \\
\hline \multirow{2}{*}{$\begin{array}{l}\text { 位点 } 4 \\
\text { 位点 } 5\end{array}$} & Pps-4 & TTCAGAGGTCTCTGACTACATGGAATCGGCAGCAAAGG \\
\hline & Pgs-5 & AGCGTGGGTCTCGGTCCACATCCATCCACTCCAAGCTC \\
\hline \multirow{2}{*}{$\begin{array}{l}\text { 位点5 } \\
\text { 位点6 }\end{array}$} & Pps-5 & TTCAGAGGTCTCTGGACTAGTGGAATCGGCAGCAAAGG \\
\hline & Pgs-6 & AGCGTGGGTCTCGCAGATAGTCCATCCACTCCAAGCTC \\
\hline \multirow{2}{*}{$\begin{array}{l}\text { 位点6 } \\
\text { 位点7 }\end{array}$} & Pps-6 & TTCAGAGGTCTCTTCTGCAATGGAATCGGCAGCAAAGG \\
\hline & Pgs-7 & AGCGTGGGTCTCGACCTCAATCCATCCACTCCAAGCTC \\
\hline \multirow{2}{*}{$\begin{array}{l}\text { 位点7 } \\
\text { 位点 } 8\end{array}$} & Pps-7 & TTCAGAGGTCTCTAGGTTTCTGGAATCGGCAGCAAAGG \\
\hline & Pgs-8 & AGCGTGGGTCTCGAGCGTTCTCCATCCACTCCAAGCTC \\
\hline \multirow{2}{*}{$\begin{array}{l}\text { 位点 } 8 \\
\text { 位点B-L }\end{array}$} & Pps-8 & TTCAGAGGTCTCTCGCTGATTGGAATCGGCAGCAAAGG \\
\hline & Pgs-L & AGCGTGGGTCTCGCTCGACGCGTATCCATCCACTCCAAGC \\
\hline 侧翼扩增引物 & $\begin{array}{l}\text { PB-R } \\
\text { PB-L }\end{array}$ & $\begin{array}{l}\text { GCGCGCGGTCTCTACCGACTAGTCACGC } \\
\text { GCGCGCGGTCTCGCTCGACGCGTATCCATC }\end{array}$ \\
\hline 载体测序引物 & $\begin{array}{l}\text { SP-L1 } \\
\text { SP-L2 } \\
\text { SP-R }\end{array}$ & $\begin{array}{l}\text { GCGGTGTCATCTATGTTACTAG } \\
\text { GTCGTGCTCCACATGTTGACC } \\
\text { TGCAATAACTTCGTATAGGCT }\end{array}$ \\
\hline 转化体阳性植株检测引物 & $\begin{array}{l}\text { Cas9-f } \\
\text { Cas9-r }\end{array}$ & $\begin{array}{c}\text { CTGACGCTAACCTCGACAAG } \\
\text { CCGATCTAGTAACATAGATGACACC }\end{array}$ \\
\hline
\end{tabular}

a) $B s a$ I 酶切末端设计成非回文序列, 可产生高效的连接(Golden Gate ligation). ACTAGT和ACGCGT分别为构建好的双元载体导入的 Spe I 和 Mlu I 切点; Spe I 为唯一切点, 可用于以“Gibson Assembly”方法 ${ }^{[3]}$ 进行第二轮克隆更多的sgRA表达盒. Mlu I 切点用于酶切分析构 建好的双元载体. 如果连接多于 8 个sgRNA表达盒, 需要自行设计更多组Pgs和Pps引物, 每组含有互补的非回文 $B s a$ I 酶切末端

Phanta Max Polymerase $0.2 \mathrm{U}$

YLgRNA-U6/U3 2 5 ng

$10 \mu \mathrm{mol} / \mathrm{L} \mathrm{U}-\mathrm{F}$ 和U\#-T\#各 $0.3 \mu \mathrm{L}$ (反应 1 )

$10 \mu \mathrm{mol} / \mathrm{L} \mathrm{gR}-\mathrm{T} \#$ 和 $\mathrm{gR}-\mathrm{R}$ 各 $0.3 \mu \mathrm{L}$ (反应2) $\mathrm{ddH}_{2} \mathrm{O}$ 补足到 $15 \mu \mathrm{L}$

25 26个PCR循环: $95^{\circ} \mathrm{C} 10 \mathrm{~s}, 58^{\circ} \mathrm{C} 15 \mathrm{~s}, 72^{\circ} \mathrm{C} 15 \mathrm{~s}$

取 $3 \sim 5 \mu \mathrm{L}$ PCR产物用 $1.5 \%$ 琼脂糖凝胶电泳检查 (反应 2 产物长度约 $140 \mathrm{bp}$ ). 如果扩增产物较弱, 也可 以继续第二轮 $\mathrm{PCR}$.

第二轮PCR. 此步骤目的是将启动子、靶点和 sgRNA构建成完整的表达盒(图3A). 表2是“金门”克隆 的第二轮通用引物, 表 3 是表 2 所示引物的使用搭配原
则, 使用时可以根据靶点数的多少合成相应的第二轮 特异性扩增的通用引物(如两靶点就合成Pps-R/Pgs-2, Pps-2/Pgs-L这两对引物即可), 并预先将通用引物对混 合成工作液(各 $10 \mu \mathrm{mol} / \mathrm{L}$ ). 第二轮PCR中各表达盒可 选择20 50 $\mu \mathrm{L}$ 反应体系( 1 个靶点 $50 \mu \mathrm{L} ; 2 \sim 3$ 个靶点各 $30 \mu \mathrm{L} ; 4$ 个或以上靶点各 $20 \mu \mathrm{L})$, 此处以 2 靶点为例, 用 $30 \mu \mathrm{L}$ 体系, 取第一轮PCR中的反应 1 和反应 2 的产物各 $1 \mu \mathrm{L}$ 加入到 $8 \mu \mathrm{L}$ 的 $\mathrm{ddH}_{2} \mathrm{O}$ 中混合稀释 10 倍, 取 $1 \mu \mathrm{L}$ 作为 第二轮 PCR的模板. 以下以 $30 \mu \mathrm{L}$ 为例.

$2 \times$ Phanta Max Buffer $15 \mu \mathrm{L}$

$10 \mathrm{mmol} / \mathrm{L}$ dNTP Mix $0.5 \mu \mathrm{L}$

Phanta Max $0.4 \mathrm{U}$ 
表 3 第二轮PCR引物的配组

\begin{tabular}{cc}
\hline 表达盒数量 & 引物组合 \\
\hline 1 & $P p s-\mathrm{R} / P g s-\mathrm{L}$ \\
2 & $P p s-\mathrm{R} / P g s-2, P p s-2 / P g s-\mathrm{L}$ \\
3 & $P p s-\mathrm{R} / P g s-2, P p s-2 / P g s-3, P p s-3 / P g s-\mathrm{L}$ \\
4 & $P p s-\mathrm{R} / P g s-2, P p s-2 / P g s-3, P p s-3 / P g s-4, P p s-4 / P g s-\mathrm{L}$ \\
5 & $P p s-\mathrm{R} / P g s-2, P p s-2 / P g s-3, P p s-3 / P g s-4, P p s-4 / P g s-5, P p s-5 / P g s-\mathrm{L}$ \\
6 & $P p s-\mathrm{R} / P g s-2, P p s-2 / P g s-3, P p s-3 / P g s-4, P p s-4 / P g s-5, P p s-5 / P g s-6, P p s-6 / P g s-\mathrm{L}$ \\
7 & $P p s-\mathrm{R} / P g s-2, P p s-2 / P g s-3, P p s-3 / P g s-4, P p s-4 / P g s-5, P p s-5 / P g s-6, P p s-6 / P g s-7, P p s-7 / P g s-\mathrm{L}$
\end{tabular}

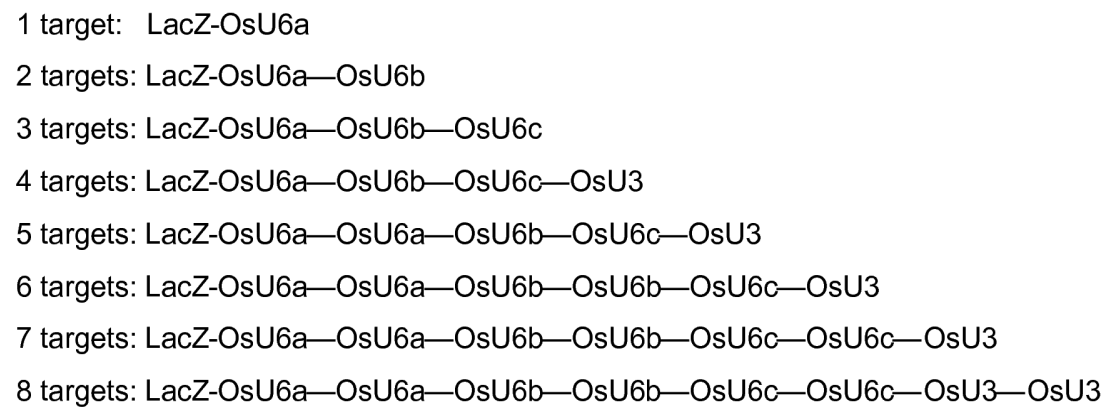

图 2 选用水稻 $\mathrm{U3} / \mathrm{U}$ 6启动子和排列原则

$10 \mu \mathrm{mol} / \mathrm{L}$ 混合通用引物 $0.5 \mu \mathrm{L}$

反应 $1+$ 反应 2 稀释液 $1 \mu \mathrm{L}$

$\mathrm{ddH}_{2} \mathrm{O}$ 补足到 $30 \mu \mathrm{L}$

25 28 PCR循环: $95^{\circ} \mathrm{C} 10 \mathrm{~s}, 58^{\circ} \mathrm{C} 15 \mathrm{~s}, 72^{\circ} \mathrm{C} 20 \mathrm{~s}$.

取 $3 \mu \mathrm{L}$ PCR产物电泳检查，并估计样品的大致 浓度.

(2) 靶点接头连接扩增法. 靶点双链接头的制备. 将接头正链和负链引物在 $0.5 \times \mathrm{TE}$ 中混合成 $0.5 \mu \mathrm{mol} / \mathrm{L}$. 约 $90^{\circ} \mathrm{C} 30 \mathrm{~s}$, 移至室温冷却完成退火. 酶切和连接(边 切边连法). 配制 $10 \mu \mathrm{L} 1 \times B s a$ I 酶切连接反应液: 在 $1 \times$ $B s a$ I 反应液中加入ATP至终浓度 $0.5 \sim 1.0 \mathrm{mmol} / \mathrm{L}$ (此 反应液对酶切和连接都有效), 加入约 $10 \mathrm{ng} \mathrm{pYLgR}$ NA-U\#质粒, $0.5 \mu \mathrm{L}$ 双链接头(最终浓度约 $0.02 \mu \mathrm{mol} / \mathrm{L}$ 或更低), 2 3 U Bsa I, 10 U T4 DNA ligase. 如果没 有ATP, 在 $1 \times B s a$ I 反应液中加入 $0.5 \sim 1.0 \mu \mathrm{L} 10 \times \mathrm{NEB}$ T4 DNA ligase buffer $(10 \times$ NEB T4 DNA ligase buffer 中含有 $10 \mathrm{mmol} / \mathrm{L} \mathrm{ATP}$ ). 用变温循环仪(或PCR仪)反应 5 个循环: $37^{\circ} \mathrm{C} 5 \mathrm{~min}, 20^{\circ} \mathrm{C} 5 \mathrm{~min}$ (没有加内切酶反应 液而只加T4 DNA ligase反应液(缺少 $\mathrm{KCl}$ 或 $\mathrm{NaCl}$ ) 不适
合内切酶反应. 双链接头浓度不要过高).

第一轮PCR: 每个 sgRNA表达盒分为 2 个PCR反应, 各 $15 \mu \mathrm{L}$ 反应体系: 取 $0.5 \mu \mathrm{L}$ 连接产物为模板, 使用 $\mathrm{U}-\mathrm{F} /$ 接头反向引物(反应 1 ), 和接头正向引物/ $\mathrm{gR}-\mathrm{R}$ (反应 2 ). 其余试剂参照 2.3 (1)第一轮PCR. 取 $4 \mu \mathrm{L}$ 产物电泳检 查. 第二轮PCR: 条件与 2.3 (1)第二轮PCR相同.

(3) PCR产物纯化. 根据第二轮各表达盒PCR片段 的浓度, 把所有表达盒片段大致等量混合, 用 PCR 产物 纯化试剂盒纯化.

\section{4 将sgRNA表达盒克隆到pYLCRISPR/Cas9 载体}

此步骤使用基于 $B s a$ I 酶切和连接的“金门”克隆 方法, 以“边切边连”法组装 $\mathrm{sgRNA}$ 表达盒到pYLCRISPR/Cas9载体 ${ }^{[5,9]}$. 也可以采用Gibson Assembly方 法组装sgRNA表达盒到pYLCRISPR/Cas9载体 ${ }^{[5]}$.

配制 $15 \mu \mathrm{L}$ 反应体系: 10× CutSmart Buffer $1.5 \mu \mathrm{L}$; $10 \mathrm{mmol} / \mathrm{LATP} 1.5 \mu \mathrm{L}$ (也可以加入 $1.5 \mu \mathrm{L} 10 \times \mathrm{T} 4 \mathrm{DNA}$ ligase buffer替代ATP); pYLCRISPR/Cas9质粒60 

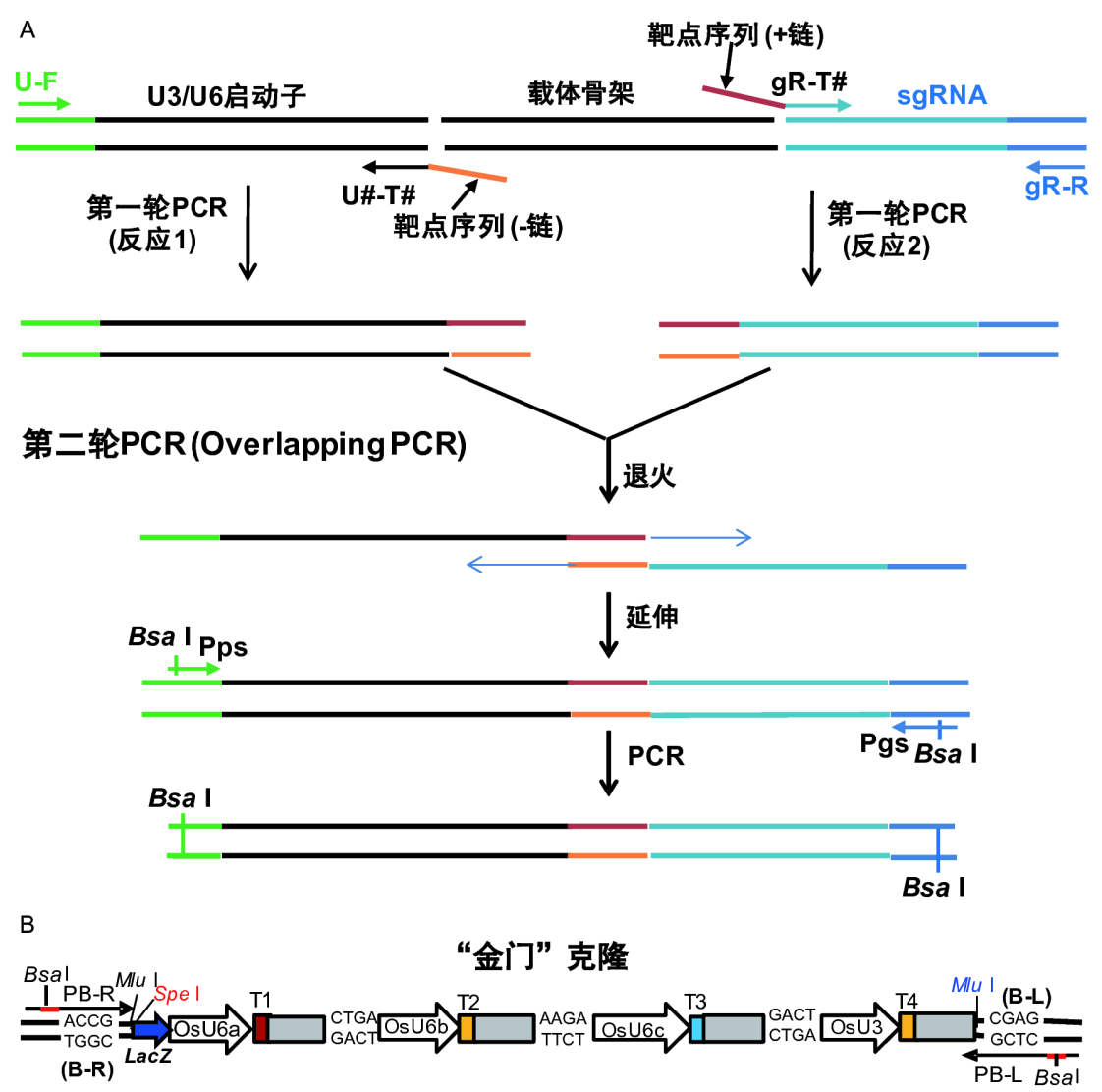

图 3 sgRNA表达盒构建原理以及靶标sgRNA表达盒与pYLCRISPR/Cas9双元载体连接原理

A: 以Overlapping PCR构建sgRNA表达盒; B: 多个靶标sgRNA表达盒与pYLCRISPR/Cas9载体的“金门”连接方法原理, 图中以四靶点为例. B-R 和B-L是经BsaI 酶切双元载体以及第一个和最后一个sgRNA表达盒产生的配对黏性末端

$80 \mathrm{ng}$; 纯化后的混合 $\operatorname{sgRNA}$ 表达盒以每个表达盒 $10 \sim 15 \mathrm{ng}$ 合计 (如 1 个靶点约 $10 \mathrm{ng} ; 2$ 个靶点共 20 30 ng; 3 个共约 $40 \sim 50 \mathrm{ng}$; 4 个共约 60 70 ng; 更多 靶点时每个片段的量适当增加, 每个表达盒最多 $15 \mathrm{ng}$, 这样载体与每种插入片段摩尔比=1:4 6); $B s a$ I-HF $10 \mathrm{U}$; T4 DNA ligase $35 \mathrm{U}$; dd $\mathrm{d}_{2} \mathrm{O}$ 补足到 $15 \mu \mathrm{L}$. 用变 温循环(可使用PCR 仪)进行边切边连反应10 15盾环 $\left(37^{\circ} \mathrm{C} 5 \mathrm{~min}, 10^{\circ} \mathrm{C} 5 \mathrm{~min}, 20^{\circ} \mathrm{C} 5 \mathrm{~min}\right)$; 最后 $37^{\circ} \mathrm{C} 5 \mathrm{~min}$.

\section{5 连接产物转化}

将连接产物滴载在悬浮于 $0.2 \times \mathrm{TE}$ 的透析膜Millipore VSWP04700上透析 15 20 min (最好在 $4^{\circ} \mathrm{C}$ 冷柜 内)进行脱盐(如果没有该透析膜, 可用乙醇沉淀纯化, $70 \%$ 乙醇洗净, 风干后溶在 $5 \mu \mathrm{L}$ 去离子水, 但这样做费 时且损耗大). 取 $1 \sim 1.5 \mu \mathrm{L}$ 透析的连接产物电激转化 $E$. coli DH10B感受态细胞(化学热激法转化也可以获得
阳性克隆, 但效率较低, 四靶点以内可以尝试使用, 有 电激仪的实验室尽量使用电激法). 虽然其他菌株也可 以用, 但DH10B更适合高效率电激转化大质粒). 电激 后加入 $1 \mathrm{~mL}$ SOC培养基, $37^{\circ} \mathrm{C}$ 培养1 $1.5 \mathrm{~h}$, 将转化细 胞涂在含卡那霉素 $(25 \mu \mathrm{g} / \mathrm{mL})$ 的LB平板培养过夜. 如 果使用了LacZ-U6/U3-gRNA表达盒, 可先在每块平板 涂上IPTG/X-gal溶液 $\left(50 \mu \mathrm{L} \quad \mathrm{ddH}_{2} \mathrm{O}+10 \mu \mathrm{L} \quad 1 \mathrm{~mol} / \mathrm{L}\right.$ IPTG $+40 \mu \mathrm{L} 20 \mathrm{mg} / \mathrm{mL}$ X-gal), 使阳性克隆产生蓝色 菌斑. 空载质粒中的负篮选标记基因 $c c d B$ 可产生毒蛋 白使大肠杆菌致死.

\section{6 阳性克隆篮选}

挑取数个蓝色(如果使用了 $L a c Z$ )菌落培养和提取 质粒, 并用 Mlu I 或 Asc I 酶切和电泳确认. 也可以用 菌落PCR进行篎选, 用灭菌牙签挑取菌落的少量菌作 为模板, 对一个或所有靶点进行PCR检测. 引物配对 
形式为: SP-R引物与第一表达盒启动子反向引物配对; 第一靶点接头正链引物与第二靶点负链引物配对；第 二靶点正链引物与第三靶点负链引物配对; 第三靶点 正链引物与第四靶点负链引物配对; 如此类推. 选 1 个 阳性克隆参照图4进行测序.

\section{7 pYLCRISPR/Cas9载体导入农杆菌}

将获得的阳性克隆提取质粒, 并电激转化农杆菌 (如EHA105)。如果要做质粒在农杆菌的稳定性检测, 从农杆菌提取质粒(农杆菌提取质粒质量较差, 只适合 做PCR), 取约2 5 ng 质粒为模板, 用所有靶点接头正向 引物和反向引物配对进行PCR, 然后电泳检测确认. 如 果表达盒结构未发生变化, 获得的农杆菌可用于植物 转化.

\section{3 转化植株靶点突变类型的检测}

\section{1 阳性植株的篮选和靶点序列的扩增与测序}

利用农杆菌等方法介导的遗传转化, 获得转化植 株后，可用Cas9检测引物Cas9-f/Cas-r（表2)进一步确 认. 目前, 在多种植物已实现了较高效率的基因编辑, 且许多二倍体植物(如水稻)在 $\mathrm{T}_{0}$ 代转化体可以产生简 单突变类型即纯合突变、双等位突变或杂合突变. 这 些类型的突变可以通过PCR扩增目的片段直接进行常 规的Sanger测序. 但是, 对于嵌合突变类型(体细胞多 种多样的等位突变), 就需要采用二代高通量测序.

为了检测阳性转化体的靶点突变情况，以及提高 靶点序列扩增的特异性和测序的质量, 建议使用 CRISPR-GE的“primerDesign”, 在“primerDesign-A”设 计靶点序列扩增和测序的引物. 先选择参考基因组, 然后在“PCR template”文本框粘贴包含靶位点的基因 组序列(包含靶点及其上下游至少 $300 \mathrm{bp}$ ), 或者在基因 号栏输入目的基因号. 再输入靶点序列(如果一个PCR 扩增区含有多个靶点, 输入第一个和最后一个靶点, 并 且第一和最后靶点应相距在 $3 \mathrm{~kb}$ 以内. 较大的区间可 分为 2 个片段扩增). 正向引物距离第一个靶点的最大 和最小的距离, 以及反向引物距离最后一个靶点的最 大和最小的距离一般默认软件的初始设定(也可以修 改). 点击“Design”运行程序，结果将列出6 10对特异 扩增引物以及 $T_{\mathrm{m}}$ 值等信息. 点击第一列的ID将会直接 显示引物和靶点在目标基因组序列的具体位置，再点
击“Generate Sequencing Primer”可显示测序引物。一 个PCR扩增片段可以包含多个靶点，但每个靶点要设 计一个相应的测序引物(正向或反向都可以). 为了获 得高质量测序(低杂信号), 要使用内部的测序引物进 行测序; 再次使用PCR扩增引物进行测序容易产生高 水平杂信号, 影响结果的判读. PCR扩增目的片段后, 将PCR产物送测序公司进行常规的Sanger测序.

\section{2 利用DSDecodeM软件自动解码测序文件}

对于二倍体植物的纯合突变和两条染色体的相同 位置插入不同单碱基的杂合突变，可直接分析测序波 峰图判断突变序列. 但杂合突变及双等位突变会产生 重叠的波峰，难以简单判别突变序列. 用本课题组开 发的“简并序列解码法”(degenerate sequence decoding, DSD)可以对含有杂合突变及双等位突变的测序文件 进行手动解码 ${ }^{[6]}$. 为了提高对测序文件解码的效率, 开 发了基于DSD原理的自动解码软件“DSDecode" ${ }^{\text {"[]] }}$ 及其 升级版“DSDecodeM",[8]，可以对大量基因组编辑测序 文件进行快速分析(适用于包括双等位突变、杂合突 变和纯合突变的解码), 获得靶位点突变序列. 在 CRISPR-GE的“DSDecodeM”的参考序列输入框粘贴 野生型参考序列(如果使用从参考基因组下载的目标 区序列，要确认其必须与所编辑品种的野生型序列完 全一致). 该参考序列必须完全覆盖整个测序文件的序 列长度, 可包含一个或多个靶点. 然后上传测序峰图文 件(ab1格式; 可同时上传最多 20 个测序文件, 可以是参 考序列所覆盖的一个相同靶点或多个不同靶点的测序 文件). 对软件设定的几个参数一般不需要更改(也可 以做适当调整). 点击“Decode”运行解码程序. 解码结 果将2条等位序列和野生型参考序列排列在一起, 并分 别给出等位序列的突变类型(碱基插入、缺失、替换). 所有测序文件的解码结果可以点击“Here”下载在一个 文件保存.

如果测序文件的质量低，可能导致软件自动解码 失败. 此时可对几个参数进行调整设置, 包括调节主 峰和杂峰的信号比值, 缩短简并序列长度, 和输入一 个靶点序列(这种情况只能对一个相同靶点测序), 重 新进行解码. 或使用半自动解码工具“SaDSDecode"[7] 进行解码. SaDSDecode需要依据DSD法从测序峰图中 人工读取针定序列和简并序列输入对应的输入框进行 解码 ${ }^{[7,8]}$. 


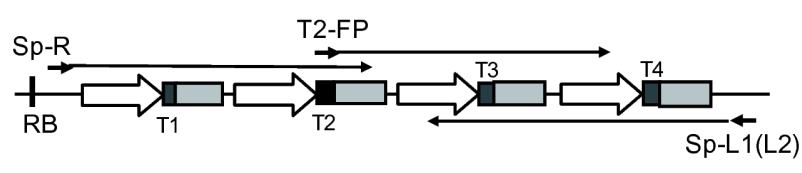

图 4 对多靶点编辑载体的sgRNA表达盒的测序策略

$\mathrm{FP}$ 为靶点双链接头连接法的正链序列引物

\section{4 常见问题和解决方法}

常见问题及其原因和解决方法如表4所示.

\section{5 结果示例}

图5显示了构建一个4靶点载体的结果.

\section{6 讨论}

在靶点设计时，建议对 1 个目标基因设计 2 个靶点 (尤其只有一个目的基因)，以降低单靶点编辑不成功 的风险. 可在可读框(open reading fream, ORF)的 $5^{\prime}$ 区 设计 2 个靶点，使之任何 1 个靶点的突变都产生基因的 功能缺失. 为了达到理想的编辑效果，应参照以下原
则设计靶点: ( i ) 目的基因的正链靶点 (5'-20N-NGG$3^{\prime} ; \mathrm{NGG}$ 为 $\mathrm{PAM}, 20 \mathrm{~N}$ 为靶点序列) 和负链靶点 ( $5^{\prime}-\mathrm{CCN}-$ $20 \mathrm{~N}-3^{\prime} ; \mathrm{CCN}$ 为PAM)的打靶效率相同，都可以设计靶 点; (ii) 靶点的 $\mathrm{GC} \%$ 尽量不要低于 $40 \%$, 靶点序列 $\mathrm{GC} \%$ 偏高 $(50 \% \sim 70 \%)$ 有较高的打靶效率; (iii) 靶点内不要 有连续 4 个以上的 T碱基, 以防RNA Pol III将其作为转 录终止信号; (iv) 为了避免或减少非特异脱靶突变, 应 进行靶点特异性分析; ( v ) 检查靶点序列与sgRNA序 列的配对情况, 靶序列与sgRNA序列产生连续配对 $8 \mathrm{bp}$ 以上会抑制其与染色体DNA靶序列结合靶点, 因 此要避免使用连续配对 $8 \mathrm{bp}$ 以上的靶点. 本实验室开 发的CRISPR-GE的“targetDesign”工具会依据上述原 则对候选靶点进行分析判别, 指出潜在的“不良”靶点.

$\mathrm{pYLCRISPR/Cas9}$ 载体系统包含从水稻克隆的 4

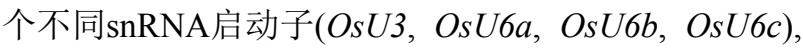
用于单子叶植物启动 $\mathrm{ggRNA}$ 表达; 从拟南芥中克隆的 4 个不同 snRNA启动子 $(A t U 3 b, A t U 3 d, A t U 6-1, A t U 6-29)$ (图1B), 用于双子叶植物启动 $\operatorname{sgRNA}$ 表达. 当构建多靶 点载体时, 为了避免在农杆菌或者植物基因组中 sgRNA表达盒之间发生同源重组，构建多靶点打靶载 体时，建议参照图2的启动子排列顺序进行排列. 由于

表 4 常见问题和解决方法

\begin{tabular}{|c|c|c|}
\hline 常见问题 & 原因 & 解决方法 \\
\hline $\begin{array}{c}\text { 在步骤2.3 (1), } 2.3 \text { (2)第一轮PCR扩 } \\
\text { 增没有目标片段带. 或者有较大的 } \\
\text { 非特异带 }\end{array}$ & $\begin{array}{c}\text { 使用错误引物或引物质量低; sgRNA质 } \\
\text { 粒纯度低并加入过多模板 }\end{array}$ & $\begin{array}{c}\text { 检查引物; 步骤 } 2.2 \text { 酶切检查sgRNA质粒的质量; 只用原来的 } \\
1 / 5 \text { 量的质粒为模板, 以减少带入的杂质(无需担心模板量不 } \\
\text { 够); 重新提取高纯度的质粒 }\end{array}$ \\
\hline $\begin{array}{c}\text { 在步骤 } 2.5 \text { 中大肠杆菌转化后没有 } \\
\text { 或很少的菌落; 或出现许多生长慢 } \\
\text { 的小菌落 }\end{array}$ & $\begin{array}{c}B s a \text { I 的活性容易下降和失活; 双元载体 } \\
\text { 质粒的质量不高导致酶切/连接后的转化 } \\
\text { 效率不高; 另外可能是感受态细胞效率低; } \\
\text { 小菌落是空载体的 } c c d B \text { 不能完全抑制住 } \\
\text { 大肠杆菌生长 }\end{array}$ & $\begin{array}{c}\text { 通过步骤2.2酶切检查 } B s a \text { I 的活性和pYLCRISPR/Cas9质粒 } \\
\text { 的质量. 建议将新购 } B s a \text { I 分装冷冻保存. 如果 } B s a \text { I 可以 } \\
\text { 消化 } s g R N A \text { 质粒(或pUC18)而不能消化pYLCRISPR/Cas9 } \\
\text { 质粒, 重新抽提pYLCRISPR/Cas9质粒; 用 } 10 \mathrm{pg} \text { pUC18转 } \\
\left.\text { 化检测感受态细胞的效率(应 }>10^{9} \text { 菌落/ } \mu \mathrm{g} \mathrm{pUC} 18\right)\end{array}$ \\
\hline $\begin{array}{l}\text { 对候选阳性克隆通过PCR和/或测 } \\
\text { 序分析, 其中部分sgRNA表达盒不 } \\
\text { 存在; 阳性克隆载体在大肠杆菌中 } \\
\text { 的序列是正确的, 但在农杆菌中后 } \\
\text { 表达盒发生了变化 }\end{array}$ & $\begin{array}{c}\text { 使用过量Bsa I /T4 DNA连接酶或过长 } \\
\text { 时间连接反应, 使不互补连接的sgRNA } \\
\text { 表达盒黏性末端被异常消化成平末端 } \\
\text { 并得以连接, 产生不完全表达盒的载 } \\
\text { 体; 步骤 } 2.3(2) \text { 的引物未正确使用; 载 } \\
\text { 体在农杆菌中发生了重组 }\end{array}$ & $\begin{array}{c}\text { 分析更多候选阳性克隆; 调整Bsa I 和T4 DNA连接酶的量, } \\
\text { 避免过度酶切/连接; 检查第二轮扩增引物对是否正确. 重 } \\
\text { 新调整U3/U6启动子的顺序 }\end{array}$ \\
\hline $\begin{array}{l}\text { 连接较多 }(5 \text { 个或以上)的 } s g R N A \text { 表 } \\
\text { 达盒的效率低, 即连接产物直接转 } \\
\text { 化后不能获得阳性克隆 }\end{array}$ & $\begin{array}{c}\text { 随着靶点的增多, 将所有靶点同 } \\
\text { 时连接上pYLCRISPR/Cas } 9 \text { 双 } \\
\text { 元载体的效率下降 }\end{array}$ & $\begin{array}{c}\text { 取步骤 } 2.4 \text { 的 } 0.5 \mu \mathrm{L} \text { 连接产物为模板, 用(表3)的引物PB-L/PB-R } \\
\text { 扩增. 凝胶电泳和回收目的片段(扩增产物可能会有非特异小片 } \\
\text { 段), 用试剂盒纯化, 再按步骤 } 2.4 \text { 进行酶切和连接; 调整载体和 } \\
\text { 插入片段的量和比例, 重新做连接 }\end{array}$ \\
\hline $\begin{array}{c}\text { 使用CRISPR-GE生成的引物错乱, } \\
\text { 及其子程序不能正常使用 }\end{array}$ & 使用浏览器的版本过低 & $\begin{array}{c}\text { 使用IE9或更高版本的IE汶览器, 如IE10, IE11等, 或者使用火 } \\
\text { 狐、谷歌等汶览器 }\end{array}$ \\
\hline $\begin{array}{c}\text { 使用DSDecodeM软件进行解码时 } \\
\text { 无结果或错误解码 }\end{array}$ & 测序质量低 & $\begin{array}{c}\text { 使用内部引物进行Sanger测序; 用软件解码前先用“Chromas”软 } \\
\text { 件打开测序文件, 检查波峰图的质量 }\end{array}$ \\
\hline
\end{tabular}




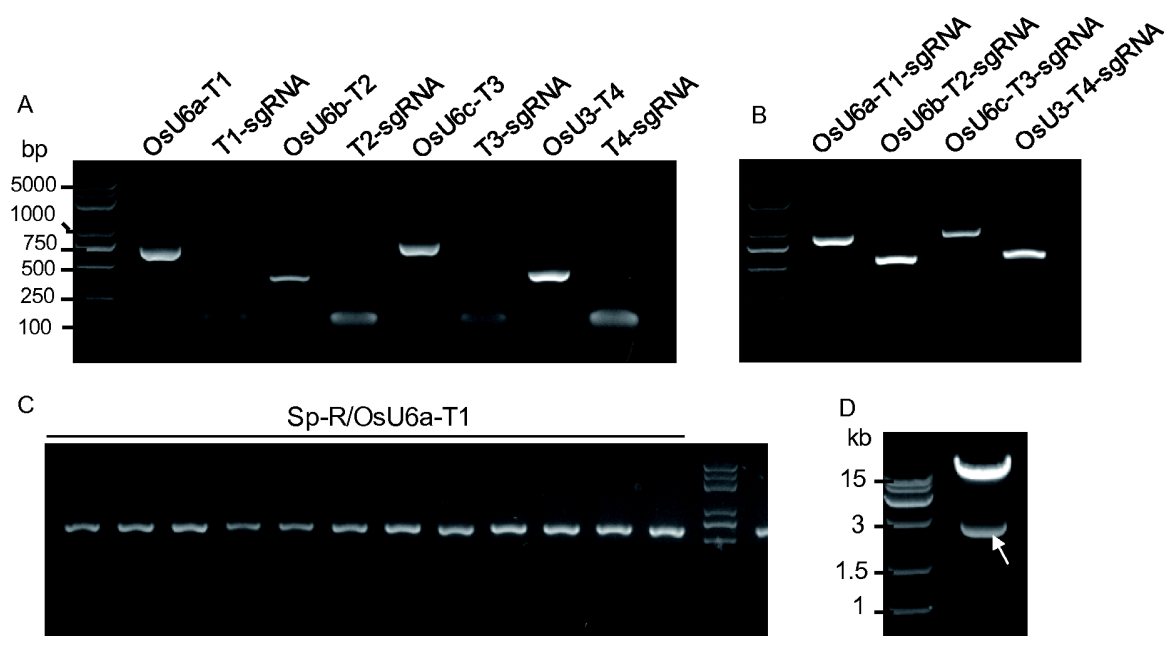

图 5 多靶点载体构建过程的结果检测

$\mathrm{A}$ : 第一轮PCR产物的琼脂糖凝胶电泳检测, 大片段为反应 1 , 小片段为反应 $2 ; \mathrm{B}$ : 第二轮PCR产物琼脂糖凝胶电泳检测; C: 以菌落PCR检测阳 性克隆. 使用引物为载体通用引物SP-R和含第一个靶点序列的OsU6a引物OsU6a-T1; D: 以 Mlu I 酶切电泳检测构建的pYLCRISPR/Cas9载体, 箭头指切出的含 4 个串联sgRNA表达盒片段

$A t U 3 d$ 很短 $(102 \mathrm{bp}$ ), 可以只使用 $A t U 3 d$ (和一个LacZAtU3d)构建多靶点编辑载体.

在构建表达盒的方法中，可选用方法1 (接头连接 法)或方法2 (overlapping PCR)往sgRNA表达盒导入靶 点序列. 方法2不需要对质粒酶切，用第一轮PCR分别 扩增出两个片段后进行第二轮overlapping PCR即可将 靶序列成功插入表达盒(图3A). 在将 $\mathrm{gRNA}$ 表达盒片段 组装到pYLCRISPR/Cas9双元载体上采用Golden Gate 克隆策略 ${ }^{[5]}$. Golden Gate克隆 ${ }^{[10]}$ 利用 II s 型内切酶 (如 $B s a$ I ) 的识别位点和切割位点不重叠的特性，可以设 计出多种不同的且非回文结构的黏性末端(256 $-16=240$ 种，减去的 16 种是回文结构). 多个要连接的 片段在连接点具有特异的互补末端可以连接, 而非连 接点的末端之间不互补不能连接(相同末端分子间也 不互补不能连接), 因此不会发生无效的连接, 使连接 反应向着目标单方向进行．本载体系统采用的Golden Gate克隆方法是将 $B s a$ I 识别位于片段上切点的外侧, 使连接成功的片段不再含 $B s a$ I 识别位点而不会被切 开. 因此可以采用简便的“边切边连”的方式, 在一个反 应中同时达到酶切和连接的目的，将多个gRNA表达 盒片段高效地同时组装到pYLCRISPR/Cas9双元载体 (图3B和5), 只需要一次大肠杆菌转化就完成多靶点载 体构建. 利用这个方法，把 8 个sgRNA表达盒同时连接 进pYLCRISPR/Cas9双元载体 ${ }^{[5]}$. 本文介绍的方法也适
用于在其他CRISPR/Cas9双元载体构建多靶点载体, 但需要对与载体连接的第一个和最后一个连接点的碱 基做相应的修改.

本套载体在单双子叶植物中都能够进行有效编 辑, 但是在不同的物种中的编辑效率有差异. 在单子叶 模式植物水稻中运用本基因编辑载体的平均编辑效率 高达 $85 \%$ 左右，在双子叶植物如拟南芥中的编辑率较 低. 其中pYLCRISPR/Cas $9 \mathrm{P}_{35 \mathrm{~s}}-\mathrm{H},-\mathrm{N},-\mathrm{B}$ 是用 $\mathrm{P}_{35 \mathrm{~S}}$ 启动子 驱动 $\operatorname{Cas} 9$ 表达, 目的是用于双子叶植物基因组编辑. 但 是, 本课题组的测试发现, 以玉米泛素基因启动子 $\mathrm{P}_{\mathrm{ubi}}$ 驱动Cas 9 表达的pYLCRISPR/Cas9Pubi-H,-N,-B在双子 叶植物中的编辑效率比使用pYLCRISPR/Cas $9 \mathrm{P}_{35 \mathrm{~s}}-\mathrm{H}$,$\mathrm{N}$,-B的高, 因为发现导入到双子叶细胞的 $\mathrm{P}_{\mathrm{Ubi}}$ 启动子的 表达水平高于 $\mathrm{P}_{35 \mathrm{~s}}$. 因此建议在大部分双子叶植物中也 优先选用pYLCRISPR/Cas9Pubi-H, -N, -B双元载体, 但 启动sgRNA表达仍然要使用拟南芥的U3/U6启动子.

pYLCRISPR/Cas9载体系统和配套的在线网络分 析工具包CRISPR-GE已在国内外获得广泛应用，并在 各个领域取得一些代表性成果: 例如，华南农业大学 庄楚雄教授课题组 ${ }^{[1]}$ 编辑水稻TMS5 基因, 并用DSDecodeM 分析靶点突变, 快速培育多个温敏不育系; 中山 大学肖仕教授课题组 ${ }^{[12]}$ 编辑拟南芥中 TRAF $1 a$ 和 $T R A F 1 b$ 基因, 研究其在细胞自噬中的作用; 西南大学 罗克明教授课题组 ${ }^{[13]}$ 编辑杨树 (Populus) ptrWRKY18 和 
$p t r W R K Y 35$ 基因, 揭示WRKY转录因子在提高锈病抵 抗力中的作用; 中国科学院华南植物园黄宏文教授课 题组 ${ }^{[14]}$ 成功编辑弥核桃 $A c P D S$ 白化基因; 此外番茄 (Lycopersicon esculentum Mill.)、香蕉(Musa nana Lour.)、油菜(Brassica napus L.)、葡萄(Vitis vinifera L.)、马铃薯(Solanum tuberosum L.)、蓝猪耳草(Torenia fournieri Linden. ex Fourn.)、棉花(Gossypium spp.) 等都已运用本套载体系统成功实现基因组编辑 ${ }^{[1520]}$.
CRISPR/Cas9 (以及CRISPR/Cpf1)技术的应用范 围将越来越广, 例如, 除了用于最常用的基因功能敲 除，还可以对敲减多拷贝重复基因的部分拷贝 ${ }^{[21] ， 以 ~}$ 及编辑目标基因的调控序列, 产生表达模式或表达水 平的变化和相应的表型变化 ${ }^{[22]}$. 基因编辑技术也在不 断发展. 本文围绕靶点选择、CRISPR/Cas9多靶点双 元载体构建, 以及对突变靶点的测序分析等提供的详 细操作方法, 为CRISPR/Cas9的应用提供了便利.

\section{参考文献}

1 Jinek M, Chylinski K, Fonfara I, et al. A programmable dual-RNA-guided DNA endonuclease in adaptive bacterial immunity. Science, 2012, 337: 816-821

2 Shan Q, Wang Y, Li J, et al. Targeted genome modification of crop plants using a CRISPR-Cas system. Nat Biotechnol, 2013, 31: 686-688

3 Li J F, Norville J E, Aach J, et al. Multiplex and homologous recombination-mediated genome editing in Arabidopsis and Nicotiana benthamiana using guide RNA and Cas9. Nat Biotechnol, 2013, 31: 688-691

4 Cong L, Ran F A, Cox D, et al. Multiplex genome engineering using CRISPR/Cas systems. Science, 2013, 339: 819-823

5 Ma X, Zhang Q, Zhu Q, et al. A robust CRISPR/Cas9 system for convenient, high-efficiency multiplex genome editing in monocot and dicot plants. Mol Plant, 2015, 8: 1274-1284

6 Ma X, Chen L, Zhu Q, et al. Rapid decoding of sequence-specific nuclease-induced heterozygous and biallelic mutations by direct sequencing of PCR products. Mol Plant, 2015, 8: 1285-1287

7 Liu W, Xie X, Ma X, et al. DSDecode: a web-based tool for decoding of sequencing chromatograms for genotyping of targeted mutations. Mol Plant, 2015, 8: 1431-1433

8 Xie X, Ma X, Zhu Q, et al. CRISPR-GE: a convenient software toolkit for CRISPR-based genome editing. Mol Plant, 2017, 10: 1246-1249

9 Ma X, Liu Y. CRISPR/Cas9-based multiplex genome editing in monocot and dicot plants. Curr Protoc Mol Biol, 2016, 115: 31.6.1-31.6.21

10 Engler C, Kandzia R, Marillonnet S. A one pot, one step, precision cloning method with high throughput capability. PLoS ONE, 2008, 3: e3647

11 Zhou H, He M, Li J, et al. Development of commercial thermo-sensitive genic male sterile rice accelerates hybrid rice breeding using the CRISPR/Cas9-mediated TMS5 editing system. Sci Rep, 2016, 6: 37395

12 Qi H, Xia F N, Xie L J, et al. TRAF family proteins regulate autophagy dynamics by modulating AUTOPHAGY PROTEIN6 stability in Arabidopsis. Plant Cell, 2017, 29: 890-911

13 Jiang Y, Guo L, Ma X, et al. The WRKY transcription factors PtrWRKY18 and PtrWRKY35 promote Melampsora resistance in Populus. Tree Physiol, 2017, 37: 665-675

14 Wang Z, Wang S, Li D, et al. Optimized paired-sgRNA/Cas9 cloning and expression cassette triggers high-efficiency multiplex genome editing in kiwifruit. Plant Biotechnol J, 2018, in press doi: 10.1111/pbi.12884

15 Yang Y, Zhu G, Li R, et al. The RNA editing factor SlORRM4 is required for normal fruit ripening in tomato. Plant Physiol, 2017, 175: 16901702

16 胡春华, 邓贵明, 孙晓玄, 等. 香蕉CRISPR/Cas9基因编辑技术体系的建立. 中国农业科学, 2017, 50: 1294-1301

17 Yang Y, Zhu K, Li H, et al. Precise editing of CLAVATA genes in Brassica napus L. regulates multilocular silique development. Plant Biotechnol J, 2018, in press doi: 10.1111/pbi.12872

18 Wang X, Tu M, Wang D, et al. CRISPR/Cas9-mediated efficient targeted mutagenesis in grape in the first generation. Plant Biotechnol J, 2018, 16: $844-855$

19 Su S, Xiao W, Guo W, et al. The CYCLOIDEA-RADIALIS module regulates petal shape and pigmentation, leading to bilateral corolla symmetry in Torenia fournieri (Linderniaceae). New Phytol, 2017, 215: 1582-1593

20 Gao W, Long L, Tian X, et al. Genome editing in cotton with the CRISPR/Cas9 system. Front Plant Sci, 2017, 8: 1364 
21 Li X, Xie Y, Zhu Q, et al. Targeted genome editing in genes and cis-regulatory regions improves qualitative and quantitative traits in crops. Mol Plant, 2017, 10: 1368-1370

22 Rodríguez-Leal D, Lemmon Z H, Man J, et al. Engineering quantitative trait variation for crop improvement by genome editing. Cell, 2017, 171: 470-480.e8

\title{
A protocol for CRISPR/Cas9-based multi-gene editing and sequence decoding of mutant sites in plants
}

\author{
ZENG DongChang $^{1}$, MA XingLiang ${ }^{1,2}$, XIE XianRong ${ }^{1}$, ZHU QinLong ${ }^{1} \&$ LIU Yao-Guang ${ }^{1}$ \\ ${ }^{1}$ State Key Laboratory For Conservation and Utilization of Subtropical Agro-Bioresources, College of Life Sciences, South China Agricultural \\ University, Guangzhou 510642, China; \\ ${ }^{2}$ Seed and Developmental Biology Program, Global Institute for Food Security, University of Saskatchewan, Saskatoon, SK S7N 4J8, Canada
}

Genome editing with CRISPR/Cas9 has become an effective tool for gene functionality dissection and crop improvement. Accordingly, we have developed a CRISPR/Cas9 vector system for robust multi-gene editing in plants. Our inventory includes six versatile binary vectors and 12 intermediary sgRNA vectors with different U3 and U6 promoters, to achieve high efficient multi-gene editing, under specific antibiotic selections in both monocot and dicot plants. Additionally, we also have developed an integrated webbased software toolkit, CRISPR-GE, to facilitate the utilization CRISPR/Cas9 in plants. In this protocol, we provide detailed procedures for using the CRISPR/Cas9 system, including target site selection, construction of CRISPR/Cas9 binary vectors, Sangersequencing-based genotyping of mutant sites, and troubleshooting for common problems.

plant genome editing, CRISPR/Cas9, targeted mutation, CRISPR-GE

doi: $10.1360 / \mathrm{N} 052018-00069$ 